\title{
Eating and drinking in rats with anterior or posterior amygdaloid lesions
}

\author{
WILLIAM L. STOLLER and RITA A. V. STOLLER \\ Indiana University at Kokomo, Kokomo, Indiana 46901
}

\begin{abstract}
Electrolytic lesions were placed bilaterally either in the anterior or in the posterior amygdala of albino rats. In terms of comparisons of lesion data with data for animals with corresponding control operations, anterior amygdaloid lesions had no significant influence on either water or overall food intake nor on body weight, while posterior amygdaloid lesions significantly increased water consumption without reliably altering food intake or body weight. It is proposed that the dorsal portion of the anterior amygdala may contain neural elements which facilitate food consumption and inhibit water intake, that the posterodorsal amygdala may contain excitatory elements for food intake, and that the middle portion of the amygdala as a whole may facilitate water intake.
\end{abstract}

In a previous study (Stoller, 1972), relatively large bilateral lesions situated in the amygdaloid complex of rats decreased water intake and produced a loss of body weight. In addition, the data indicated that such lesions might reduce food consumption. Although histological examination of the amygdala and neighboring structures revealed a few instances of damage to the posterior striatum (striatum = caudatoputamen nucleus), a subsequent investigation (Stoller, 1974) suggested that striatal injury had exerted relatively little, if any, influence on the previous findings.

Information has accumulated indicating that amygdaloid influences on eating and drinking are region dependent. Lesions in the dorsomedial amygdala, for example, have resulted in aphagia (Fonberg, 1969), whereas damage involving the basolateral region has produced hyperphagia (Green, Clemente, \& De Groot, 1957). Collier and Gault (1969) observed both aphagia and adipsia as a result of lesions involving the medial nuclei. Grossman and Grossman (1963) found that, in rats, water intake was reduced by lesions in the anterior or middle portion of the ventral amygdala, but was increased by posteroventrally placed lesions. More recently Box and Mogenson (1975) observed that lesions in the dorsal region of the central amygdaloid nucleus of the rat produced aphagia and adipsia, whereas lesions in the ventral posterior portion of the lateral amygdala resulted in hyperphagia.

In view of such findings the present study sought to extend the analyses of the previous investigations in this laboratory (Stoller, 1972, 1974) to a comparison of the possible effects of anterior and posterior amygdaloid lesions on eating and drinking.

This study was partially supported by funds received from the Office of Research and Advanced Studies at Indiana University. Reprints may be obtained from William L. Stoller, Indiana University at Kokomo, 2300 South Washington Street, Kokomo, Indiana 46901.

\section{METHOD}

\section{Subjects}

The subjects were 32 male albino rats obtained from the Holtzman Company (Madison, Wisconsin). The animals were about 120 days old at the beginning of experimentation and had no prior experimental history. They were housed in individual cages.

\section{Surgery and Histology}

Bilateral electrolytic lesions were produced in the amygdala with the aid of a Baltimore stereotaxic instrument. A 1-mA anodal current was applied for $12 \mathrm{sec}$ through a steel needle (.5 mm diam) insulated except for the tip. For anteriorly placed lesions the coordinates were $2.0 \mathrm{~mm}$ posterior to bregma, $4.0 \mathrm{~mm}$ lateral to the midline, and $8.0 \mathrm{~mm}$ below the dura mater. The coordinates for posteriorly placed lesions differed from these only in that the AP position was $3.8 \mathrm{~mm}$ posterior to bregma. The incisor bar was located $8 \mathrm{~mm}$ below the interaural line. Animals that received control operations underwent exactly the same surgical procedures as the animals in the corresponding lesion groups, except that no current was applied. All animals were maintained under sodium pentobarbital anesthesia (Nembutal, $60 \mathrm{mg} / \mathrm{kg}$ IP) during surgery.

At the end of the experiment, the rats were killed with sodium pentobarbital and the brains were fixed in $10 \%$ Formalin. Representative 40-micron coronal sections obtained through the use of a frozen tissue technique were mounted and stained with cresyl violet. The lesions were evaluated by comparing the sections with plates in the König and Klippel (1963) atlas.

\section{Procedure}

Eight animals were randomly assigned to each of four treatments: anterior amygdaloid lesion (AN-AM), anterior amygdaloid control (AN-AM-CON), posterior amygdaloid lesion (POS-AM), and posterior amygdaloid control (POS-AM-CON). Following surgery an animal was given food (Purina Rat Chow) and tap water ad lib during a 4-day period that was allowed for recovery. Over the next 5 days the subject was adapted to a $2 \frac{1}{4}-\mathrm{h}$ daily eating period, which was established for an operant conditioning phase of the study. The conditioning data are not directly relevant to the present paper and consequently will not be considered. Water was freely accessible to the animal in its home cage for about $231 / 4 \mathrm{~h}$ each day following the postoperative recovery period.

Beginning on the 10th postoperative day, data on daily food and water intake and on body weight were recorded for a period 
of 20 days. Home-cage intake measures were provided by a difference method (Stoller, 1972). The amount of 45-mg food pellets (P. J. Noyes Company) consumed during a daily operant training session was added to the home-cage food-intake value to give the total daily food consumption measure for each animal. Body weight was determined $1 \frac{1 / 4}{h}$ before the eating period. All measurements for food, water, and body weight were made to the nearest gram. The data were evaluated with analyses of variance.

\section{RESULTS}

\section{Food and Water Intake}

Figures 1 and 2 show food and water intake relative to preoperative body weight over the 20-day observa-

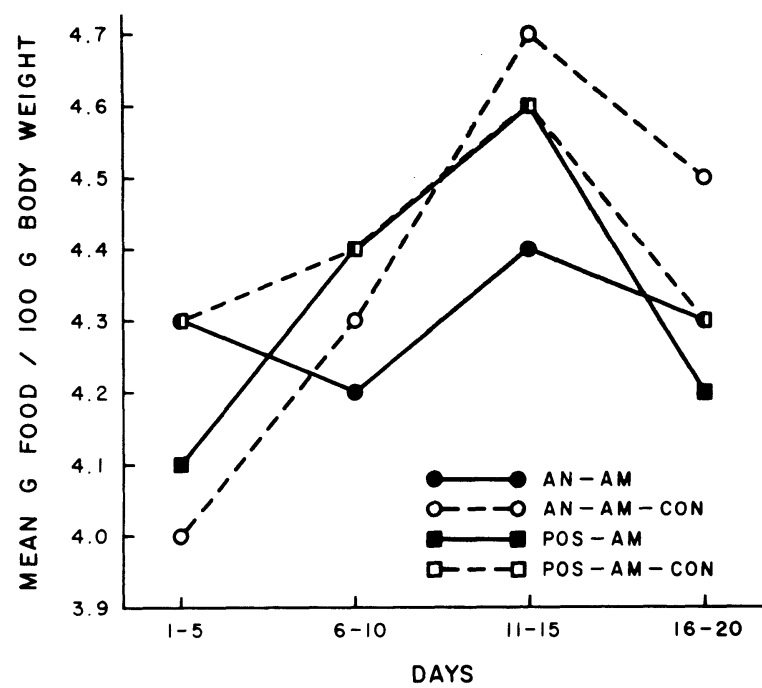

Figure 1. Food intake relative to preoperative body weight for 20-day period beginning on 10th postoperative day. (Abbreviations: $\mathbf{A N}-\mathbf{A M}=$ anterior amygdaloid lesion, $\mathbf{A N}-\mathbf{A M}-\mathbf{C O N}=$ anterior amygdaloid control, $\mathrm{POS}-\mathrm{AM}=$ posterior amygdaloid lesion, POS-AM-CON = posterior amygdaloid control.)

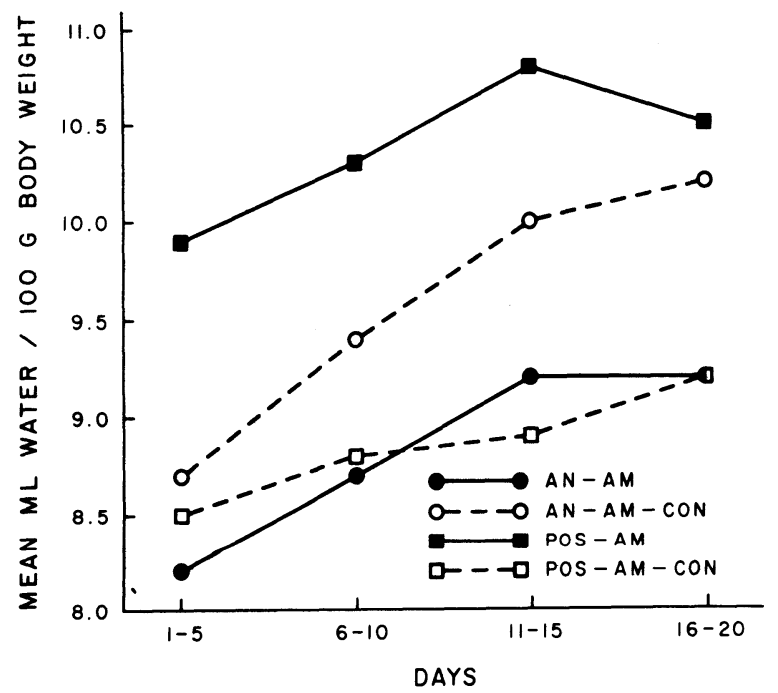

Figure 2. Water intake relative to preoperative body weight for 20-day period beginning on 10th postoperative day. (Abbreviations as for Figure 1.) tion period. Analyses of variance on data for the entire period yielded a significant treatment effect for POS-AM vs. POS-AM-CON with respect to water consumption $[F(1,14)=4.8, p<.05]$. It can be seen in Figure 2 that the animals with the posterior amygdaloid lesions consistently consumed more water per $100 \mathrm{~g}$ of body weight than did the corresponding control subjects. On the other hand, the treatment effects for AN-AM vs. AN-AM-CON and POS-AM vs. POS-AM-CON with respect to food consumption were nonsignificant, as was the treatment effect for AN-AM vs. AN-AM-CON in the case of water intake $(\mathrm{Fs}<1.0)$.

Days effects were significant for both the food measures $[F(3,42)=4.0, p<.05 ; F(3,42)=6.7, p<.001$; anterior and posterior lesion analyses, respectively] and water measures $[\mathrm{F}(3,42)=17.3, \mathrm{p}<.001 ; \mathrm{F}(3,42)=$ $4.0, \mathrm{p}<.05$; anterior and posterior lesion analyses, respectively]. An examination of Figures 1 and 2 reveals a general increase in the measures over the observation period. In the instance of the food-intake data for AN-AM vs. AN-AM-CON, the Treatments by Days interaction was significant $[\mathrm{F}(3,42)=3.0, \mathrm{p}<.05]$. Figure 1 shows that mean food consumption per $100 \mathrm{~g}$ of body weight for the animals with anterior amygdaloid lesions exceeded that for the corresponding controls on the first block of 5 days, but was less than the control intake over the remaining blocks. The Treatments by Days interactions for the remaining ingestive data fell far short of significance $(\mathrm{Fs}<1.0)$.

\section{Weight Changes}

Figure 3 shows changes in body weight relative to preoperative weight over the 20-day period. Although the subjects in both lesion groups displayed reduced weight in relation to the corresponding controls, the treatment effects did not reach statistical significance

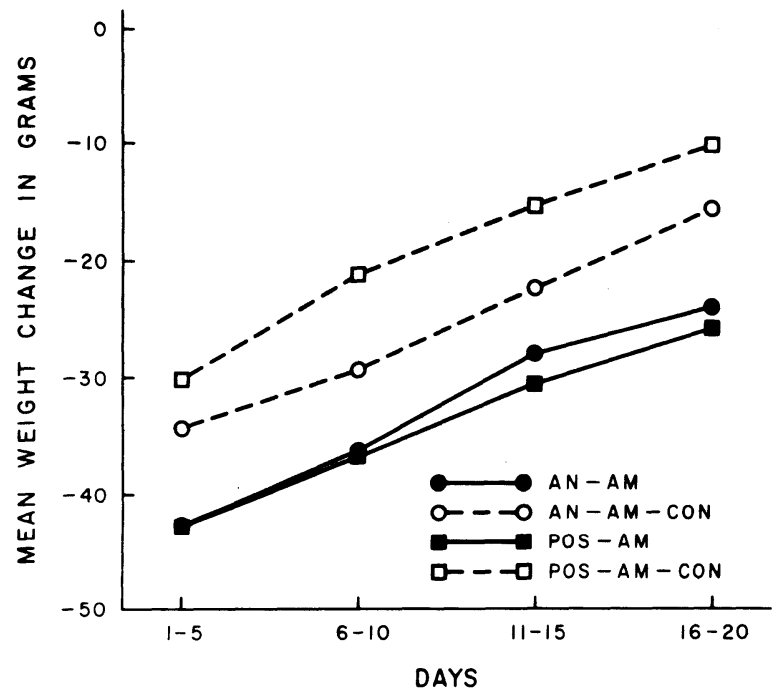

Figure 3. Changes in body weight relative to preoperative level for 20 -day period beginning on 10 th postoperative day. (Abbreviations as for Figure 1.) 
$(\mathrm{Fs}=1.2$ and 4.2 for $\mathrm{AN}-\mathrm{AM}$ vs. AN-AM-CON and POS-AM vs. POS-AM-CON, respectively; $d f$ s $=1$ and 14 ; ps $>.05)$. In contrast, the days effects were highly significant ( $F s=2.3 .8$ and 39.7 for anterior and posterior lesion analyses, respectively; $\mathrm{dfs}=3$ and $42 ; \mathrm{ps}<.001$ ). An examination of Figure 3 shows that all groups gained steadily in body weight. The Treatments by Days interactions were nonsignificant $(\mathrm{Fs}<1.0)$.

\section{Lesion Placements}

The anterior lesions typically included nearly all of the anterior amygdaloid area. At the AP plane where the lesions tended to be greatest, corresponding approximately to A $6650 \mu$ in the König and Klippel (1963) atlas, the damage extended about $1.5 \mathrm{~mm}$ in the mediolateral dimension and $1.7 \mathrm{~mm}$ dorsoventrally. The lesions were generally about $1.5 \mathrm{~mm}$ in anteroposterior extent. Damage to structures lying outside of the amygdaloid region was slight.

The posterior lesions typically included much of the corticomedial and basolateral regions, which displayed essentially the same degree of injury. Damage tended to be maximal at an AP plane corresponding approximately to A $3900 \mu$ in the König and Klippel system, the lesion measurements being about 1.5 and $1.3 \mathrm{~mm}$ in the mediolateral and dorsoventral dimensions, respectively. The lesions extended $1.5 \mathrm{~mm}$ anteroposteriorly. Damage to extra-amygdaloid structures was negligible.

\section{DISCUSSION}

In the present study anterior amygdaloid lesions had no significant influence on either water or overall food intake nor on body weight, while posterior amygdaloid lesions significantly increased water consumption without reliably altering food intake or body weight.

The design of the study appears to have been favorable for the demonstration of experimental effects. The methods of data collection and analy sis were similar to those employed previously (Stoller, 1972). The observation period was nearly identical to that for which water intake and body weight were found to be reduced as a consequence of the larger amygdaloid lesions (Stoller, 1972). Further, the 21/4-h daily eating period does not seem to have been too restrictive, in view of pilot observations which indicated little food consumption after the first $1 \frac{1}{2} \mathrm{~h}$ of the period. The lesions themselves, although less extensive than the amygdaloid lesions of the initial study, were larger than those described in the second experiment of Grossman and Grossman (1963) as having apparently permanent effects on food and water intake.

Grossman and Grossman (1963) proposed that a "very diffuse inhibitory mechanism" (p. 765) regulating food intake may exist throughout the ventral amygdala. For the regulation of water intake, on the other hand, these investigators suggested the existence of an excitatory mechanism in the anteroventral part of the amygdala and an inhibitory mechanism in the posteroventral amygdala. Since, in the present study, the anterior lesions involved both the dorsal and ventral portions of the amygdala, the negative findings for ingestive effects in the case of the anterior placements might conceivably be attributed to neural elements in the dorsal amygdala which facilitate food consumption and inhibit water intake. In the instance of the posterior lesions, which also involved the dorsal and ventral amygdala, the results suggest in the same manner that the dorsal amygdala may contain excitatory elements for food intake. The findings are consistent with the Grossman and Grossman (1963) evidence for an inhibitory water regulation mechanism in the posterior amygdala.

Previously, water intake was reduced by relatively large lesions which were situated essentially in the anterior-middle region of the amygdala (Stoller, 1972). In view of the anterior lesion results of the current investigation, it thus appears that, as a whole, the middle portion of the amygdala may facilitate water intake.

\section{REFERENCES}

Box, B. M., \& Mogenson, G. J. Alterations in ingestive behaviors after bilateral lesions of the amygdala in the rat. Physiology and Behavior, 1975, 15, 679-688.

Collier, B. D., \& GAulT, F. P. Aphagia and adipsia following lesions of the amygdala. Psychonomic Science, 1969, 17, 41-42.

FoNBERG, E. Effects of small dorsomedial amygdala lesions on food intake and acquisition of instrumental alimentary reactions in dogs. Physiology and Behavior, 1969, 4, 739-743.

Green. J. D., Clemente, C. D., \& De Groot, J. Rhinencephalic lesions and behavior in cats. Journal of Comparative Neurology, 1957, 108, 505-545.

Grossman, S. P., \& Grossman, L. Food and water intake following lesions or electrical stimulation of the amygdala. American Journal of Physiology, 1963, 205, 761-765.

König, J. F. R., \& KLIPPEL, R. A. The rat brain. Baltimore: Williams \& Wilkins, 1963.

STOLLER, W. L. Effects of septal and amygdaloid lesions on discrimination, eating and drinking. Physiology and Behavior, 1972, 8, 823-828.

STOLLER, W. L. Food, water and saccharin solution intake in rats with posterior striatal lesions. Physiology and Behavior, 1974, 13, 599-602.

(Received for publication September 26, 1977.) 